\title{
A Survey on Congestion Control in Wireless Sensor Networks
}

\author{
K. Sumathi \\ Dept of CSE \\ EBET Group of Institutions \\ Kangayam, India
}

\author{
M. Venkatesan, PhD \\ Dept of CSE \\ K.S.R Institute for Engg \& Tech \\ Thiruchengode, India
}

\begin{abstract}
Congestion in wireless sensor networks creates a lot of issues like packet collision, buffer overflow, queuing delay and many to one data transmission scheme. This leads to degrade the quality of service parameters like packet delivery ratio, end to end delay and Average energy consumption of the wireless nodes. In this work is used to describe some of the congestion control mechanisms used in the WSNs and classifies them into four major categories traffic rate control, resource management, traffic rate and resource management and priority based congestion control. The comparative analysis is used to compare the popular congestion control protocols with each other in terms of congestion detection, congestion notification and its advantages and disadvantages.
\end{abstract}

\section{Keywords}

Wireless sensor networks, Congestion detection, Congestion notifications, Congestion control.

\section{INTRODUCTION}

A wireless sensor network consists of huge number of sensors, which are responsible for monitoring physical or environmental conditions like temperature, sound, vibration, pressure at various locations. In the recent centuries numbers of applications of WSN [1] are increased vigorously. Some of the applications are health monitoring, industry production, home automation and environmental monitoring. These sensors are small in size as well as limited processing and computing resources. Congestion is occurred in the sensor network at the time of a sensor node is carrying much amount of traffic than it can handle. It will creates a series issue in the network such as queuing delay, packet loss, increases response time and decrease the throughput. To handle this situation in an effective manner a number of congestion control protocol should be used. Any congestion control mechanism follows three steps i) Congestion detection ii) congestion notification iii) congestion control.

\section{CONGESTION DETECTION MECHANISMS}

In literature, the authors considered more number of metrics for detecting congestion in a network such as packet loss, buffer size, channel load and delay. This paper is used to describe some of the parameters and Figure1 contains congestion detection metrics [2].

\subsection{Packet loss}

Packet loss is an important metric to detect the congestion in the network. The packet loss is occurred in the network in the following manner.

Near source: Sensor nodes are deployed in a dense region will generate a hot spot near a source at unexpected events. During this time the congested node generate back pressure congestion notification to the source; the source will adjust its traffic rate consequently. The local de-synchronization of source and resources is also an effective technique to reduce congestion in a network.

Near sink: Sensor nodes are deployed in a sparse region will generate a hot spot in a sensor field but farther from source, near a sink. To handle this situation very effectively localized back pressure and packet dropping techniques can be followed. Use of multiple sinks uniformly scattered across the sensor field is an alternative solution for the above said problem.

Medium collision: In a certain area, many nodes start its transmission at a same time creates interference of data leads to packet loss in the network. By using explicit local synchronization among neighbors and reduce this type losses. But this type of situation cannot be eliminated completely because non-neighboring nodes are still interfering with transmission.

Buffer over follow: Generally a queue or buffer is used to hold the packets at the time of transmission. A buffer can received more number of packets than it can transmit, at that time buffer over follow will occur leads to packet loss in the network.

\subsection{Buffer Size}

Buffer size is a second important metric to detect the congestion in the network. It can be measured in 2 ways as follows.

Buffer limit: Each and every node in the WSN has limited buffer to hold the data to transmit. A buffer size is can be used as threshold, if incoming packets cross buffer's threshold leads to packet loss.

Remaining Buffer: During transmission of packet the buffer capacity is periodically tested and finds the remaining buffer length out of the overall size or the difference between the remaining buffer and the traffic rate can be used as the best congestion indicators.

\subsection{Delay}

Delay is a third important metric to detect the congestion in the network. It can be measured in 2 ways as follows. 


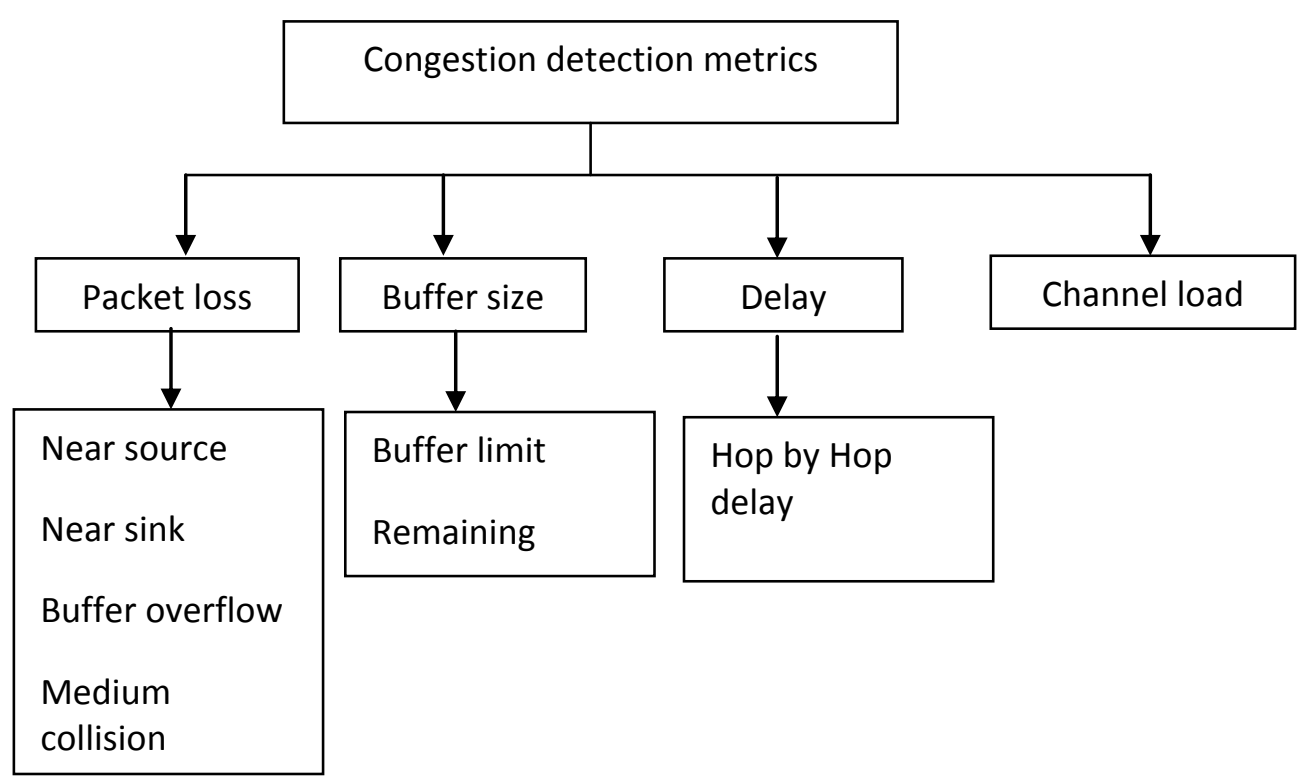

Figure1. Congestion Detection metrics

Hop by Hop delay: The packets are generated at the sender and forwarded to the next hop. The time is taken for transfer a packet from one hop to another hop. The one hop delay is also an important factor for detecting congestion in a network because it includes packet waiting time in a buffer, collision resolution and packet transmission time at the MAC layer. The one hop delay is varied according to the channel load and buffer capacity.

End to End delay: The packets are generated at the sender and successful forwarded to the end point or receiver. The time is taken for transfer a packet from one end hop to another end hop.

\section{Channel load}

Channel load is a fourth important metric to detect the congestion in the network

Channel load is the ratio between either channel is busy for successful transmission of packets collision to the total time period. If collision increases then the number of packets is dropped, consequently the buffer occupancy is decreased misleads to inference of the absence of congestion. The buffer

state is used as congestion indicator here but to get accurate congestion detection the combination of both queue length and channel load should be used.

\section{CONGESTION NOTIFICATION}

After identification of congestion, it should be intimated to the upstream nodes to take a necessary action and control congestion. Congestion information can be propagated by using explicit or implicit congestion notification. Some

protocols notify the congestion by setting congestion notification bit in the packet header.

\subsection{Explicit congestion notification:}

In this type the control packets are generated at the time of congestion and which are forwarded to either source or sink to intimate congestion level. Since additional control packet generate an additional load to the network. A fewer number of congestion control mechanism follow this method.

\subsection{Implicit congestion notification:}

Unlike explicit method, this method does not give any additional load to the congested node. During congestion the congested nodes implicitly creates piggybacking information and inform its congestion level to its upstream nodes. In some cases ACK packets are used to indicate the congestion state. A larger number of congestion control mechanism follow this method.

\section{CONGESTION CONTROL ALGORITHMS IN SENSOR NETWORKS}

After notification of congestion the source or sink node take a necessary action to control the congestion in a network otherwise it leads to buffer overflow, packet loss, delay and resource wastage. To control congestion in a network number of strategies can be followed such as traffic rate control, resource management or combination of traffic control and resource management and priority based congestion control mechanisms which are depicted in the Figure2.

\subsection{Traffic rate control:}

In the traffic rate control technique, congestion is controlled by reducing number of packets injected into wireless sensor networks. It is divided into additive increase multiplicative decrease AIMD or a rate based method. In AIMD verify networks available bandwidth and slowly increase size of the congestion window. During congestion the protocol decreases the congestion window significantly. In the following session is used to describing some of the traffic rate based congestion control methods.

\section{CODA}

C.Y. Wan and his team introduced Congestion detection and Avoidance in a sensor networks. This is an energy efficient congestion control protocol. In this method buffer length and channel load metrics are used to detect the congestion. At the 
time of congestion the congested node notifies its congested situation to its upstream nodes through open loop hop by hop back pressure to decrease its traffic rate. At the same time sink generates an ACK through closed loop to source to reduce its data generation rate. The CODA [4] consumes an additional energy to transfer an ACK and backpressure.

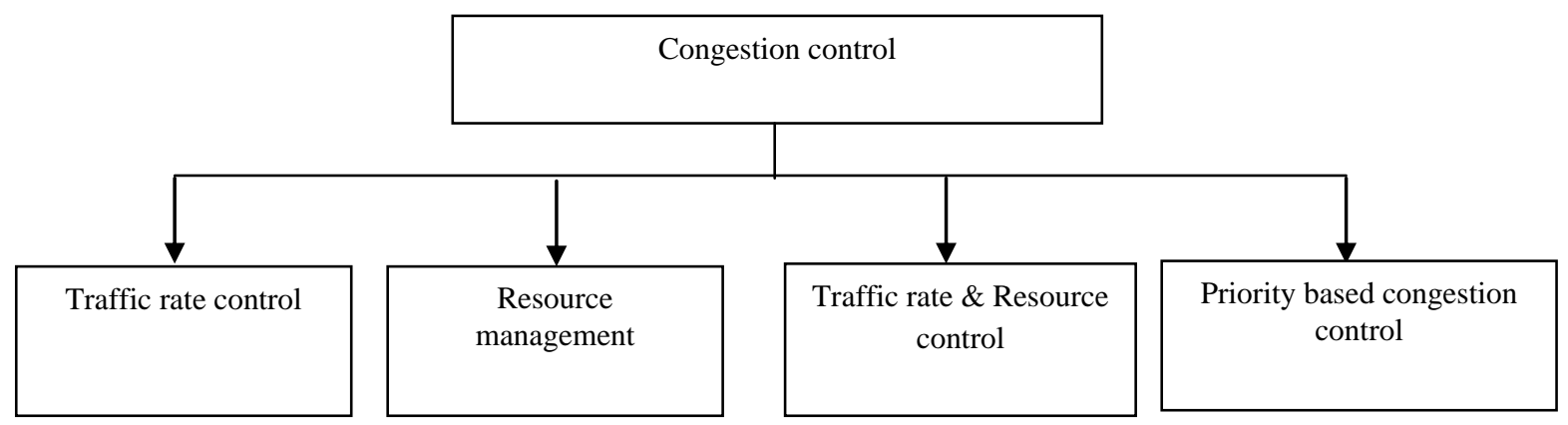

Figure2. Congestion control techniques

The CODA uses AIMD concept which leads to packet loss. The CODA depends on unidirectional congestion control which increases timelines and reduces reliability of the network.

\section{2. $A R C$}

A. Woo and his colleague introduced Adaptive Rate Control (ARC).In ARC [5] there is no congestion detection or notification mechanism instead it uses AIMD concept. In which an intermediate node increases its data transfer rate by a constant rate ' $a$ ' at the time of successful packet forwarding by its parent node otherwise multiplies its sending rate by a factor ' $b$ '.In ARC two independent traffic set is maintained for giving fairness to the traffic such as factor ' $a$ ' is called source traffic and ' $b$ ' is called transit traffic. In ARC there is no congestion detection or implicit or explicit congestion notification mechanisms. The ARC rate adjustment scheme is also introduces a packet loss.

\section{3. $C C F$}

Ee and R. Bajcsy introduced congestion control and fairness protocol is a distributed and scalable mechanism for many to one routing in WSNs.Here congestion are detected based on packet service time and congestion controls through reduction of rate of traffic. CCF [6] control congestion in a hop by hop manner and each node adjusts its traffic rate based on its available service rate and child node number. In CCF rate adjustment is a function of packet service time leads to low utilization of sensor nodes and creates significant packet error rate. It does not includes current buffer capacity leads to queuing delay and buffer overflow as well as the number of retransmission of packet is increased.

\section{4. $C A D A$}

Fang et al introduced CADA [7]- Congestion Avoidance Detection and Alleviation in WSNs.In this algorithm the congestion is detected by an aggregation of buffer occupancy and channel load. Here considered the growing rate of buffer occupancy to a certain limit, after that limit they are considered as congested. For the moment the packet delivery ratio is decreased considerably at the time of local channel load is reaches the maximum channel utilization it leads to congestion in the channel .Congestion occurs due to the traffic emergence it will be detected instantaneously by the hotspot depends on a combination of buffer occupancy and channel utilization.
Moreover, congestion is alleviated reactively by either dynamic traffic control or source rate regulation according to specific hotspot scenarios.CADA optimizes throughput, energy consumption and average end-to-end delay.

\section{ECODA}

Enhanced congestion detection and avoidance was introduced to detect congestion in WSN by using dual buffer threshold and weighted buffer difference. ECODA [8] uses the packet scheduler to detect the priority of the packet and during congestion low priority packets are dropped. ECODA is used to handle both transient and persistent congestion in a smooth manner. The transient congestion is control by implicit hop by hop back pressure method and reduction of data rate at source is used to control persistent congestion.

\subsection{Resource management}

The traffic control method is not suitable for event based application. To overcome this method an alternative method called resource control. Here when the network is congested data packets follow alternative paths, which are not congested, in order to be forwarded to sink. This method has the advantage that traffic control is avoided and all data packets have a great opportunity to reach the sink. At the same time special care needs to be taken in order to meet the performance requirement like packet travel time, avoidance of loops etc. In the following session is used to describing some of the resource management based congestion control methods.

\section{TARA}

J. Kang et al introduced Topology Aware Resource Adaptation protocol which is used to adapt the network's additional resources at the time of congestion .A graph coloring strategy is used to find the needed topology for the adaption of additional resources. TARA[9] considers both buffer capacity and channel load to detect the congestion. TARA utilizes distributor and merger, distributor is used to distribute the traffic between the original path and detour path and merger merges two flows. During congestion, traffic is deflected from the hotspot through the distributor node along the detour and reaches the merger node. The main drawback of this protocol is not suitable for large scale sensor networks. 


\section{HTAP}

C. Sergiou proposed Hierarchical Tree Alternative Path protocol which is scalable and distributed for reducing the congestion and assuring reliable data transmissions. It is a hop by hop algorithm and implicitly informing congestion to other nodes. By using resource control mechanism the congestions are mitigated by choosing an alternative path from source to the sink, which are formed by using unused nodes of the network. Through simulation they prove that use of these nodes leads to balance the energy consumption and avoid the creation of holes in the network and prolonging the network life time.HTAP[10] consists of four different schemes topology control, hierarchical tree creation, alterative path creation and handling of powerless nodes. In topology control scheme, the nodes belonging to the topologies are updated in the neighbor table. In hierarchical tree creation, the tree is created and source node is act as a tree's root. The connection is created between transmitter and receiver using 2-way handshake method and which is used to indicate the congestion level from receiver node to transmitter node. In alternative path production, the transmitter node selects a node from its neighbor table that has no congestion. In the final part, if the battery of a sensor node is deplete, the neighbor table of will be updated.

\section{CONSISE}

Vedantham introduced an adaptive and explicit rate control protocol is called as Congestion Control from Sink to Sensor (CONSISE).It deals the congestion control in a different manner from sink to sensors instead of sensor to sink. In CONSISE [11] the congestion is detected by the sensor node and they adjust their sending rate based on the congestion level in end of each period. Upstream nodes informed the congestion level to downstream nodes by using explicit congestion notification. The downstream nodes adjust its data rate based on information received through explicit notification. It uses the available resources in a efficient way with minimum overhead.

\subsection{Traffic rate control and Resource management}

This is a hybrid method to combine advantages of both traffic control and resource management. It is an application dependent. The traffic control method is suitable for transient congestion where as resource control method is applied in the permanent congestion control situation. In the following session is used to describing some of the traffic rate based and resource management based congestion control methods.

\section{TALONet}

Huang proposed TALONet [12] as a Power-Efficient GridBased Congestion Avoidance Scheme Using Multi detouring Technique. It consists of 3 schemes such as maintain 3 different power levels to reduce congestion in the data link layer, to avoid buffer overflow buffer management techniques followed and to handle heavy traffic multi-path detouring techniques used. It also consists of three phases such as network formation phase, data dissemination phase and framework updating phase. In the network formation phase each and every node receives a control packet from sink and known its location and build an imaginary square grids. The nodes in the grids are called TOLEN or normal nodes. In the data dissemination phase the data are disseminated by the TOLEN nodes. After receiving control packets from sink the networks are updated in the network updating phase. It gives a better result in terms of power consumption and packet drops compared to TARA.

\section{TCEER}

Arpita Chakraborty and their team introduced Trust integrated Congestion Aware Energy Efficient Routing algorithm. In this malicious nodes are isolated from the data path. The node potential is computed based on the trust. By using fuzzy logic controller the congestion status identified with the input of residual energy and distance of the node from the base station. The source node is responsible for initiating the routing process by selecting the node with high potential in its one hop radio range. The node present in one hop radio range is light weight but more energy efficient. It is suitable for larger WSN. Through simulation results the author show that TCEER [13] is $25 \%$ more efficient than other protocol in terms of number of rounds and network performance. The major drawback in TCEER algorithm is, it has been tested against small networks.

\subsection{Priority based congestion control:}

In this section is used to discuss some of the priority based congestion control protocols in wireless sensor network. Also compare their mechanisms with one another.

\section{PCCP}

Wang introduced Priority based congestion control protocol. In PCCP the author gives an equal fairness to each and every sensor nodes in a multi hop WSN. In PCCP [14] different priority indexes are maintained such as a node with higher priority utilizes higher bandwidth and node with higher data rate uses more bandwidth. It is used to measure the congestion degree as the ratio between packet arrival time and its service time along with its priority index and hop by hop cross layer based congestion control mechanisms followed. The implicit congestion notifications are done with the help of piggybacking the congestion information along the header of the data packet and avoid use of unnecessary control packets. In PCCP the energy efficiencies optimized. The PCCP gives lot of advantages such as lower buffer utilization, low packet utilization and low delay. The main draw back in PCCP as there is no packet recovery mechanisms followed.

\section{2. $D P C C$}

Heikalabad introduced dynamic prediction based congestion control algorithm. The DPCC [15] is used to dynamically predict the congestion in a sensor node and fairly broad casting the traffic to the entire network. It is used to increase the throughput and reduce the number of packet loss with low overhead. In DPCC three steps are followed backward and forward node selection, predictive congestion detection and dynamic priority based rate adjustment. In the forward and backward node selection, the forward and backward nodes are selected for data handling. In the second and third steps are used to detect the congestion and through implicit notification the rate of the packets are adjusted and congestion are eliminated at the MAC layer.

\section{PASCCC}

Mian Ahmad Jan introduced an energy-efficient application specific clustering congestion control protocol. This protocol is implemented based on queuing model. In this congestion are detected according to the mobility and heterogeneity of the nodes. This protocol is mainly used in fire detection, home 
automation and related applications. In which congestion spots are detected and also detect the node causes the congestion. Here each incoming packets are prioritized as high priority and low priority packets. During congestion low priority packets are discarded. Through an experimental result the author show that the PASCCC [16] significantly improves lifetime of the network, energy consumption and data delivery between $\mathrm{CHs}$ and BS.The PASCCC has two disadvantages, it is an application dependent and there is no packet recovery.

\section{COMPARATIVE ANALYSIS OF PROTOCOLS}

In this section is used to compare the different congestion control protocols with each other with respect to the following factors: congestion detection metrics, congestion notification, advantages and disadvantages. Table 1, 2, 3, and 4 demonstrates these comparison according to their congestion controlling techniques such as traffic rate control(Table 1),resource management(Table 2),traffic rate and resource management(Table 3 ) and priority based congestion control (Table 4).

\section{CONCLUSION AND FUTURE WORK}

From the discussion, it was known that congestion control is one of the major as well as unpredictable events of the WSNs. The congestion in the network leads to energy waste, throughput reduction and number of packet loss results in network's performance degradation. This paper is used to describe a survey of some of the popular congestion control protocols in wireless sensor networks. This paper clearly describes about the congestion detection metrics, congestion notification and congestion control mechanisms in a detailed manner. The comparative study shows that the pros and cons of the popular congestion control protocols. The main objective of this work as improve the life time of the WSNs by the selecting of the best congestion control mechanisms for the given application. The future work of this paper mainly focused on designing energy efficient and trust based congestion control protocol which also includes one or more features like decentralized, self adapted, distributed, scalable, autonomous, generalized and secured congestion control strategies.

\section{ACKNOWLEDGMENTS}

Our thanks to the anonymous reviewers for their valuable comments and suggestions.

\section{REFERENCES}

[1] I. Akyildiz, W. Su, Y. Sankarasubramaniam, and E. Cayirci. A survey on sensor networks. Communications Magazine, IEEE, 40(8):102 - 114, Aug 2002.

[2] Mohamed Amine Kafi, Djamel Djenouri, Jalel Ben Othman, Abdelraouf Ouadjaout,, Nadji Badache," Congestion Detection Strategies in Wireless sensor Networks: A Comparative Study with Test bed Experiments", The 5th International Conference on Emerging Ubiquitous Systems and Pervasive Networks EUSPN-2014.

[3] Ali Ghaffari,"Congestion control mechanisms in wireless sensor networks: A survey" Journal of Network and Computer Applications March 2015.
[4] C.Y. Wan, A.T. Campbell and S.B. Eisenman, "CODA: congestion detection and avoidance in sensor networks", in Proceedings of the 1st international Conference on Embedded Networked Sensor Systems: (SenSys '03), ACM, 2003, pp. 266-279.

[5] A. Woo and D. E. Culler, "A Transmission Control Scheme for Media Access in Sensor Networks," in Proceedings of the 7th annual International Conference on Mobile Computing and Networking (Mobi-Com'01), 2001, pp. 221-235.

[6] C. T. Ee and R. Bajcsy, "Congestion Control and Fairness for Many-to-One Routing in Sensor Networks," in SenSys '04: Proceedings of the 2nd international Conference on Embedded Networked Sensor Systems, 2004, pp. 148-161.

[7] W.W. Fang, J.M. Chen, L. Shu, T.S. Chu and D.P. Qian, "Congestion avoidance, detection and alleviation in wireless sensor networks", Journal of Zhejiang UniversitySCIENCE C, Vol. 11, no.1, 2010, pp. 63-73.

[8] L. Tao and F. Yu, "ECODA: enhanced congestion detection and avoidance algorithm for multiple class of traffic in sensor networks", IEEE Transactions on Consumer Electronics, Vol. 56, no. 3, 2010, pp. 1387-1394.

[9] J. Kang, Y. Zhang, and B. Nath, "TARA: Topology-Aware Resource Adaptation to Alleviate Congestion in Sensor Networks," IEEE Transactions on Parallel and Distributed Systems, vol. 18, no. 7, pp. 919-931,2007.

[10] C. Sergiou, V. Vassiliou, and A. Paphitis, "Hierarchical Tree Alternative Path (HTAP) Algorithm for Congestion Control in Wireless Sensor Networks," Ad Hoc Network., vol. 11, no. 1, pp. 257-272, Jan. 2013.

[11] Vedantham R, Sivakumar R, Park S-J. Sink-to-sensors congestion control. Ad Hoc Netw 2007;5(4):462-85.

[12] J.-M. Huang, C.-Y. Li, and K.-H. Chen, "TALONet: A Power-Efficient Grid-Based Congestion Avoidance Scheme Using Multi-Detouring Technique in Wireless Sensor Networks," in Wireless Telecommunications Symposium, 2009, pp. $1-6$.

[13] CHAKRABORTY ET AL Trust Integrated Congestion Aware Energy Efficient Routing for Wireless Multimedia Sensor Networks (TCEER). Journal of Computing and Information Technology - CIT 23, 2015, 2, 95-109.

[14] C. Wang, K. Sohraby, V. Lawrence, B. Li, and Y. Hu, "Priority-based congestion control in wireless sensor networks," International Conference on Sensor Networks, Ubiquitous, and Trustworthy Computing,vol. 1, pp. 22-31, 2006.

[15] Heikalabad SR, Ghaffari A, Hadian MA, Rasouli H. DPCC: dynamic predictive congestion control in wireless sensor networks. IJCSI Int $\mathbf{J}$ Comput Sci Issues 2011;8(1):472-7.

[16] Mian Ahmad Jan, Priyadarsi Nanda, Xiangjian He , Ren Ping Liu ," PASCCC: Priority-based application-specific congestion control clustering protocol", Computer Networks 74 (2014) 92-102. 


\section{APPENDIX}

Table 1. Popular Traffic rate control congestion control protocols and its features

\begin{tabular}{|c|l|l|l|l|}
\hline Protocol & \multicolumn{1}{|c|}{$\begin{array}{c}\text { Congestion detection } \\
\text { metrics }\end{array}$} & $\begin{array}{c}\text { Congestion } \\
\text { notification }\end{array}$ & \multicolumn{1}{|c|}{ Advantages } & \multicolumn{1}{|c|}{ Disadvantages } \\
\hline CODA & $\begin{array}{l}\text { Buffer length and channel } \\
\text { load }\end{array}$ & Explicit & Energy efficient & Non reliable, Packet loss \\
\hline ARC & Buffer length & Implicit & $\begin{array}{l}\text { Avoid use of control } \\
\text { messages, fairness }\end{array}$ & Packet loss \\
\hline CCF & $\begin{array}{l}\text { Packet service time, queue } \\
\text { length }\end{array}$ & Implicit & Distributed and scalable & $\begin{array}{l}\text { Queuing delay, buffer } \\
\text { overflow and increasing } \\
\text { retransmission }\end{array}$ \\
\hline CADA & $\begin{array}{l}\text { Buffer length and channel } \\
\text { load }\end{array}$ & Implicit & $\begin{array}{l}\text { Improves throughput, energy } \\
\text { consumption }\end{array}$ & \begin{tabular}{l} 
Unfairness \\
\hline ECODA
\end{tabular} \\
$\begin{array}{l}\text { Dual Buffer threshold and } \\
\text { weighted buffer difference }\end{array}$ & Implicit & $\begin{array}{l}\text { Energy efficient, reduced } \\
\text { delay, better QoS }\end{array}$ & Lack of packet recovery \\
\hline
\end{tabular}

Table 2.Popular Resource management congestion control protocols and its features

\begin{tabular}{|l|l|l|l|l|}
\hline \multicolumn{1}{|c|}{ Protocol } & \multicolumn{1}{|c|}{$\begin{array}{c}\text { Congestion } \\
\text { detection metrics }\end{array}$} & $\begin{array}{c}\text { Congestion } \\
\text { notification }\end{array}$ & \multicolumn{1}{|c|}{ Advantages } & \multicolumn{1}{|c|}{ Disadvantages } \\
\hline TARA & $\begin{array}{l}\text { Buffer occupancy } \\
\text { and channel load }\end{array}$ & Explicit & $\begin{array}{l}\text { Provides trustworthiness } \\
\text { satisfaction and energy } \\
\text { conservation. Improves } \\
\text { performance compared to the past }\end{array}$ & $\begin{array}{l}\text { Not suitable for large scale } \\
\text { network. }\end{array}$ \\
\hline HTAP & Buffer occupancy & Implicit & Simple,sclable, and distributed & It is not energy efficient. \\
\hline CONSISE & $\begin{array}{l}\text { Buffer occupancy } \\
\text { and channel load }\end{array}$ & Explicit & $\begin{array}{l}\text { Improved performance with } \\
\text { minimum overhead. }\end{array}$ & $\begin{array}{l}\text { Congestion is controlled } \\
\text { from sink to sensors instead } \\
\text { of sensor to sink. }\end{array}$ \\
\hline
\end{tabular}

Table3.Popular Traffic and Resource management congestion control protocols and its features

\begin{tabular}{|l|l|l|l|l|}
\hline \multicolumn{1}{|c|}{ Protocol } & \multicolumn{1}{|c|}{$\begin{array}{c}\text { Congestion } \\
\text { detection } \\
\text { metrics }\end{array}$} & \multicolumn{1}{|c|}{$\begin{array}{c}\text { Congestion } \\
\text { notification }\end{array}$} & \multicolumn{1}{|c|}{ Advantages } & \multicolumn{1}{|c|}{ Disadvantages } \\
\hline TALONet & $\begin{array}{l}\text { Buffer } \\
\text { occupancy }\end{array}$ & Explicit & $\begin{array}{l}\text { Amount of power consumption } \\
\text { is low. Number of packet loss } \\
\text { is reduced. }\end{array}$ & $\begin{array}{l}\text { There is no congestion control } \\
\text { and back pressure algorithm. }\end{array}$ \\
\hline TCEER & $\begin{array}{l}\text { Buffer capacity } \\
\text { and Remaining } \\
\text { Energy }\end{array}$ & Explicit & $\begin{array}{l}\text { Suitable for large scale WSN.It } \\
\text { gives more security. }\end{array}$ & $\begin{array}{l}\text { TCEER has been tested against } \\
\text { only small networks. }\end{array}$ \\
\hline
\end{tabular}

Table4.Popular Priority based congestion control protocols and its features

\begin{tabular}{|c|l|l|l|l|}
\hline Protocol & $\begin{array}{c}\text { Congestion } \\
\text { detection metrics }\end{array}$ & $\begin{array}{c}\text { Congestion } \\
\text { notification }\end{array}$ & \multicolumn{1}{|c|}{ Advantages } & \multicolumn{1}{c|}{ Disadvantages } \\
\hline PCCP & $\begin{array}{c}\text { Buffer occupancy, } \\
\text { Packet arrival and } \\
\text { service time }\end{array}$ & Implicit & $\begin{array}{l}\text { Fairness, energy efficient, } \\
\text { low delay, low buffer } \\
\text { occupancy }\end{array}$ & There is no packet recovery \\
\hline DPCC & $\begin{array}{c}\text { Queue occupancy } \\
\text { and channel load }\end{array}$ & Implicit & $\begin{array}{l}\text { Increase throughput, low } \\
\text { control overhead }\end{array}$ & Not energy efficient \\
\hline PASCCC & Buffer occupancy & Implicit & $\begin{array}{l}\text { Mobility. Increase network } \\
\text { life time and data } \\
\text { transformation. }\end{array}$ & $\begin{array}{l}\text { Application dependent. } \\
\text { There is no packet recovery. }\end{array}$ \\
\hline
\end{tabular}

